\section{Estudo da mortalidade de recém-nascidos internados na UTI neonatal do Hospital Geral de Caxias do Sul, Rio Grande do Sul}

\section{Newborn mortality study in the neonatal intensive care unit of Caxias do Sul General Hospital, Rio Grande do Sul}

Breno Fauth de Araújo 1

Ana Cristina d' A. Tanaka 2

José Mauro Madi 3

Helen Zatti 4

\author{
1 Serviço de Neonatologia. Hospital Geral. Universidade de Caxias \\ do Sul. Av. Prof. Antônio Vignolli, 255. Bairro Petrópolis. Caxias do \\ Sul, Rio Grande do Sul, Brasil. CEP: 95.070-561 E-mail: \\ bfaraujo@terra.com.br \\ 2 Departamento de Saúde Materno-Infantil. Faculdade de Saúde \\ Pública. Universidade de São Paulo, São Paulo, SP, Brasil \\ 3 Serviço de Obstetrícia. Hospital Geral. Universidade de Caxias do \\ Sul. Caxias do Sul, RS, Brasil \\ 4 UTI Neonatal. Hospital Geral. Hospital Geral. Universidade de \\ Caxias do Sul. Caxias do Sul, RS, Brasil
}

\begin{abstract}
Objectives: to determine causes and variables related to newborn deaths of a neonate intensive care unite (ICU) in the Southern region of Brazil.

Methods: a descriptive study involving 2.247 newborns followed up until discharge date. Maternal variables were analyzed, as well as of the newborn and pregnancy, delivery and medical assistance variables. Univariate analysis and multiple logistic regression were used to relate the variables studied with death occurrence.

Results: 184 deaths occurred, corresponding to a $8.2 \%$ lethality rate. Newborn mortality variables were the following: weight of $<1.500 \mathrm{~g}$ corresponding to $29.3 \%$, with $61.3 \%$ under $1.000 \mathrm{~g}$ and $5.6 \%$ over 1.000g. Low weight and premature newborns held a death risk four times higher than newborns weighing $\geq 2.500 \mathrm{~g}$ or born at pregnancy term. Death related variables were weight of $<2.000$ grams, Apgar at 5' $<4$, no prenatal medical care, the need of delivery room resuscitation procedures and mechanic ventilation during hospitalization. Deaths basic causes were malformations (25.6\%), maternal hypertension (17.9\%) and maternal infections (12.0\%).

Conclusions: many of the deaths occurred because of the poor medical care offered to pregnant women and newborns, a challenge that need to be faced by neonatologists, obstetricians and the government.
\end{abstract}

Key words Infant, newborn, Infant mortality, Risk factors

\section{Resumo}

Objetivos: conhecer as causas e variáveis relacionadas com o óbito de recém-nascidos (RN) de uma UTI neonatal de referência na região Sul do Brasil.

Métodos: estudo descritivo envolvendo $2.247 \mathrm{RN}$ acompanhados até a alta. Foram analisadas variáveis maternas, do RN e variáveis da gestação, parto e atendimento. Foi utilizada a análise univariada e a regressão logística múltipla para relacionar as variáveis estudadas com o óbito.

Resultados: ocorreram 184 óbitos, com uma letalidade de $8,2 \%$. A mortalidade dos $R N$ com peso $<1.500 \mathrm{~g}$ foi de 29,3\%, sendo de 61,3\% abaixo de $1.000 \mathrm{~g}$ e 5,6\% acima de $1.000 \mathrm{~g}$. Os RN com baixo peso e os prematuros apresentaram um risco de morrer quatro vezes maior do que os $R N$ com peso $\geq 2.500 \mathrm{~g}$ ou a termo. As variáveis relacionadas ao óbito foram o peso $<2.000$ gramas, $o$ Apgar no $5^{\prime}<4$, a ausência de pré-natal, a necessidade de reanimação em sala de parto e a ventilação mecânica durante a internação. As principais causas básicas de óbito foram as malformações (25,6\%), hipertensão materna $(17,9 \%)$ e as infecções maternas $(12,0 \%)$.

Conclusões: muitos óbitos ocorreram por falhas no atendimento da gestante e do RN, desafio que precisa ser enfrentado por neonatologistas, obstetras e o poder público.

Palavras-chave Recém-nascido, Mortalidade infantil, Fatores de risco 


\section{Introdução}

Um bom indicador da qualidade de vida de uma população é o coeficiente de mortalidade infantil. Essa taxa possui dois componentes importantes, que são o de mortalidade neonatal e o de infantil tardio ou pós-neonatal. Quanto mais desenvolvido for o país maior é o peso do componente neonatal sobre a mortalidade infantil, pois as condições sócio-ambientais fazem com que a mortalidade pós-neonatal se reduza.

No Brasil, nas últimas décadas, este fenômeno também vem ocorrendo e a mortalidade infantil teve um declínio forte a partir dos anos 80 . Porém nessa década o componente pós-neonatal foi superior ao neonatal: 27,8 por mil nascidos vivos e 20,3 por mil nascidos vivos respectivamente. No final da década de 90 houve a inversão esperada onde a mortalidade neonatal passou a ser quase duas vezes maior que a pós-neonatal, isso é, a mortalidade infantil em 2000 foi de 21,3 por mil nascidos vivos, sendo a neonatal de 13,6 por mil nascidos vivos e a pós-neonatal de 7,7 por mil nascidos vivos. 1

Tal fato ocorreu devido ao controle da doença diarréica, melhoria das moradias, maior acesso ao saneamento básico, campanhas de vacinação, - que reduziram as doenças infecciosas no primeiro ano de vida - e a melhoria das condições de vida da população mais carente. ${ }^{2}$

A mortalidade neonatal, entretanto, tem permanecido relativamente estável por ser resultante de uma estreita e complexa interação entre fatores biológicos, sociais, econômicos, políticos, demográficos e de assistência à saúde, que a tornam um componente de difícil controle. Isso faz com que seja, atualmente, a responsável por $75 \%$ das oito milhões de mortes de crianças com menos de 1 ano em todo o mundo. ${ }^{3}$

A importância cada vez maior do componente neonatal na constituição da mortalidade infantil tem gerado inúmeros estudos sobre as causas e fatores determinantes das mortes nesse período. ${ }^{4-6}$

$\mathrm{O}$ peso de nascimento e a idade gestacional são os fatores isolados mais importantes relacionados ao óbito neonatal. Araújo et al. ${ }^{7}$ encontraram um risco de óbito seis vezes maior entre os recém-nascidos com peso menor do que $2500 \mathrm{~g}$ e/ou idade gestacional menor do que 37 semanas.

A incidência de partos prematuros, que durante muitos anos permaneceu entre 8 e $10 \%$ na maioria dos países desenvolvidos, nos últimos anos vem aumentando gradativamente devido ao aumento no número de gestações múltiplas causadas por tratamentos de infertilidade. Nos Estados Unidos 8 a porcentagem de nascimentos prematuros aumentou de 9,4\%, em 1981, para 12\% em 2002 e com isso aumentou, também, o número de recém-nascidos de baixo peso de $6,7 \%$ para $7,8 \%$ no mesmo período.

Entretanto, em países em desenvolvimento como o Brasil predominam, ainda, os partos prematuros e de crianças com baixo peso ao nascer, desencadeados por más condições sociais e econômicas, infecções e um deficiente atendimento pré-natal.

Além do baixo peso de nascimento e da prematuridade, são fatores importantes de óbito, nesses países, as causas preveníveis e relacionadas com a qualidade de atenção perinatal, como a anóxia e as infecções neonatais, ${ }^{9}$ diferentemente dos países desenvolvidos, onde predominam as malformações congênitas. 10

O objetivo deste estudo foi conhecer as causas de óbito e as principais variáveis relacionadas com o óbito dos recém-nascidos internados em uma unidade de terapia intensiva neonatal de referência que atende pacientes de baixa renda na região sul do Brasil.

\section{Métodos}

A população de estudo foi constituída por todos os recém-nascidos internados na UTI Neonatal do Hospital Geral (HG), Caxias do Sul, RS, no período de março de 1998 a março de 2004 e que preencheram os critérios de inclusão e exclusão do estudo. O Hospital Geral é um hospital público, universitário que atende somente pacientes do Sistema Único de Saúde (SUS).

O serviço de Obstetrícia e Neonatologia do HG é referência para gestantes de risco da região Nordeste do Estado que engloba 50 municípios. Esse fato resulta no recebimento de um grande número de $\mathrm{RN}$ e gestantes de risco oriundas de outras cidades, o que contribui, atualmente, com $40 \%$ da ocupação de leitos na UTI neonatal e explica a alta taxa de internação na UTI dos nascidos no hospital.

Foram incluídos no estudo todos os RN internados na UTI neonatal do HG no período do estudo, sendo excluídos os recém-nascidos com peso inferior a 500 gramas e/ou idade gestacional inferior a 24 semanas e os RN sem informação sobre o peso e/ou idade gestacional, resultando em um total de 2247 recém-nascidos (RN).

Trata-se de um estudo descritivo, sendo todos os recém-nascidos acompanhados desde a internação até a saída da UTI neonatal, que ocorreu com a alta para o domicílio, transferência para outro hospital ou pela ocorrência de óbito. 
Foram analisadas as características da mãe e do $\mathrm{RN}$, as variáveis relacionadas com a gestação, parto e atendimento do RN durante a internação e a causa básica do óbito, classificada de acordo com a definição da Classificação Internacional de Doenças (CID 10). ${ }^{11}$

" Causa básica de óbito é a doença ou lesão que iniciou a cadeia de acontecimentos patológicos que conduziram diretamente à morte."

Os dados foram coletados pelos autores da pesquisa a partir do prontuário da gestante e do $\mathrm{RN}$ internado na UTI Neonatal e armazenados em um banco de dados do programa Epi-info, versão 6.0 .

Todas as análises estatísticas foram feitas usando o pacote estatístico SPSS (Statiscal Package for the
Social Science, versão 12).

A análise univariada foi realizada para relacionar as variáveis do estudo com o desfecho de interesse (óbito do RN). Para comparar médias de amostras independentes foi realizado o teste $t$ e para variáveis dicotômicas utilizou-se o teste $\chi^{2}$ bicaudal. As variáveis estatisticamente significativas na análise univariada compuseram o modelo para a análise multivariada (regressão logística múltipla). Adotou-se um nível de significância de $\alpha=5 \%$ e um erro $\beta=80 \%$.

\section{Resultados}

Durante os seis anos do estudo nasceram no Hospital Geral 10.060 recém-nascidos, com uma média de

Tabela 1

Número de nascidos vivos (NV), número e percentagem de óbitos e Odds ratio (OR) dos recém-nascidos do Hospital Geral segundo o peso de nascimento. Caxias do Sul, Rio Grande do Sul, 1998 a 2004.

\begin{tabular}{lrrrr}
\hline Peso (gramas) & Nascidos Vivos & & Óbitos & OR bruta $($ IC95\%) \\
\cline { 2 - 5 } & $\mathrm{n}$ & $\mathrm{n}$ & $\%$ & \\
\hline $500-749$ & 41 & 34 & 82,9 & $150,0(59,0-381,4)$ \\
$750-999$ & 65 & 31 & 47,7 & $28,1(14,4-54,9)$ \\
$1000-1249$ & 114 & 27 & 23,7 & $9,5(5,1-17,9)$ \\
$1250-1499$ & 131 & 11 & 8,4 & $2,8(1,3-6,1)$ \\
$1500-1999$ & 389 & 24 & 6,2 & $2,0(1,1-3,7)$ \\
$2000-2499$ & 400 & 16 & 4,0 & $1,2(0,6-2,5)$ \\
$2500-2999$ & 417 & 22 & 5,3 & $1,7(0,9-3,2)$ \\
$3000-3999 *$ & 625 & 19 & 3,0 & 0,001 \\
4000 e mais & 65 & 0 & - & 0,01 \\
Total & 2247 & 184 & $8,2)$ & 0,1 \\
\end{tabular}

* Categoria de referência

Tabela 2

Número de nascidos vivos (NV), número e percentagem de óbitos e Odds ratio (OR) dos recém-nascidos do Hospital Geral segundo a idade gestacional. Caxias do Sul, Rio Grande do Sul, 1998 a 2004.

\begin{tabular}{|c|c|c|c|c|c|c|}
\hline \multirow[t]{2}{*}{ Idade gestacional (semanas) } & \multirow{2}{*}{$\frac{\text { Nascidos Vivos }}{n}$} & \multicolumn{2}{|c|}{ Óbitos } & \multirow[t]{2}{*}{ OR bruta ( IC 95\%) } & \multirow{2}{*}{\multicolumn{2}{|c|}{$p$}} \\
\hline & & $\mathrm{n}$ & $\%$ & & & \\
\hline $24-27$ & 51 & 33 & 64,7 & $47,6(24,7-91,5)$ & $<0,001$ & \\
\hline $28-30$ & 125 & 49 & 39,2 & $16,7(10,4-26,9)$ & $<0,001$ & \\
\hline $37-41 *$ & 1126 & 41 & 3,6 & 1,0 & - & \\
\hline$\geq 42$ & & 20 & 1 & 5,0 & $0,7(0,1-5,5)$ & 0,5 \\
\hline Total & 2247 & 184 & 8,2 & & & \\
\hline
\end{tabular}

* Categoria de referência 
Número de nascidos vivos (NV), número e percentagem de óbitos e Odds ratio (OR) dos recém-nascidos do Hospital Geral segundo variáveis maternas e neonatais. Caxias do Sul, Rio Grande do Sul, 1998 a 2004.

\begin{tabular}{|c|c|c|c|c|c|}
\hline \multirow{2}{*}{$\begin{array}{l}\text { Variáveis } \\
\text { (maternas e neonatais) }\end{array}$} & \multirow{2}{*}{$\frac{\text { Nascidos Vivos }}{n}$} & \multicolumn{2}{|c|}{ Óbitos } & \multirow[t]{2}{*}{ OR bruta (IC95\%) } & \multirow[t]{2}{*}{$p$} \\
\hline & & $\mathrm{n}$ & $\%$ & & \\
\hline \multicolumn{6}{|l|}{ Idade mãe } \\
\hline$<15$ anos & 30 & 4 & 13,3 & $1,7(0,6-5,0)$ & 0,3 \\
\hline$\geq 15$ anos & 2054 & 167 & 8,1 & - & \\
\hline \multicolumn{6}{|l|}{ Escolaridade } \\
\hline Ausente & 29 & 2 & 6,9 & $0,9(0,2-4,0)$ & 1,0 \\
\hline Presente & 570 & 114 & 7,3 & - & \\
\hline \multicolumn{6}{|l|}{ Pré-natal } \\
\hline Não & 112 & 29 & 25,9 & $4,4(2,8-7,0)$ & $<0,001$ \\
\hline Sim & 1754 & 128 & 7,3 & - & \\
\hline \multicolumn{6}{|l|}{ Corticóide/mãe } \\
\hline Não & 1543 & 98 & 6,4 & $0,4(0,3-0,6)$ & $<0,001$ \\
\hline Sim & 632 & 82 & 13,0 & - & \\
\hline \multicolumn{6}{|l|}{ Local do parto } \\
\hline \multicolumn{6}{|l|}{ RN $2500 \mathrm{~g}$} \\
\hline Outro & 216 & 18 & 8,3 & $3,3(1,7-6,2)$ & $<0,001$ \\
\hline Hospital Geral & 864 & 23 & 2,7 & - & \\
\hline \multicolumn{6}{|l|}{ Tipo de parto } \\
\hline Normal & 1237 & 87 & 7,0 & $0,6(0,5-0,9)$ & 0,02 \\
\hline Cesárea & 909 & 90 & 9,9 & & - \\
\hline \multicolumn{6}{|l|}{ Sexo } \\
\hline Masculino & 1216 & 98 & 8,1 & $0,9(0,7-1,3)$ & 0,8 \\
\hline Feminino & 1009 & 84 & 8,3 & - & \\
\hline \multicolumn{6}{|l|}{ Reanimação em SP } \\
\hline Sim & 528 & 95 & 18,0 & $5,5(3,8-8,0)$ & $<0,001$ \\
\hline Não & 1129 & 43 & 3,8 & - & \\
\hline \multicolumn{6}{|l|}{ Apgar 1' } \\
\hline $0-3$ & 380 & 71 & 18,7 & $3,7(2,6-5,1)$ & $<0,001$ \\
\hline $4-10$ & 1707 & 99 & 5,8 & - & \\
\hline \multicolumn{6}{|l|}{ Apgar 5' } \\
\hline $0-3$ & 62 & 25 & 40,3 & $9,0(5,2-15,4)$ & $<0,001$ \\
\hline $4-10$ & 2096 & 146 & 7,0 & - & \\
\hline \multicolumn{6}{|l|}{ Uso de oxigênio } \\
\hline Sim & 817 & 101 & 12,4 & $1,5(1,1-2,3)$ & 0,01 \\
\hline Não & 525 & 43 & 8,2 & - & \\
\hline \multicolumn{6}{|l|}{ Uso de ventilação } \\
\hline Sim & 379 & 132 & 34,8 & $30,4(17,7-52,1)$ & 0,001 \\
\hline Não & 928 & 16 & 1,7 & & \\
\hline$<34$ sem & 523 & 116 & 22,2 & $6,8 \quad(4,9-9,4)$ & $<0,001$ \\
\hline$>34$ sem. & 1724 & 68 & 3,9 & - & \\
\hline \multicolumn{6}{|l|}{ Peso RN } \\
\hline $500-1999 g$ & 740 & 127 & 17,2 & $5,2 \quad(3,7-7,2)$ & $<0,001$ \\
\hline$\geq 2000 \mathrm{~g}$ & 1507 & 57 & 3,8 & & \\
\hline
\end{tabular}

* Categoria de referência 
1676 nascimentos por ano. O número de recémnascidos internados na UTI neonatal no mesmo período foi de $2.695 \mathrm{RN}$, sendo que $2.081(77,2 \%)$ RN nasceram no hospital e $614(22,8 \%)$ vieram transferidos de outros hospitais. Em relação aos nascidos no $\mathrm{HG}$, a taxa de internação na UTI neonatal foi de $20,7 \%$. Os recém-nascidos prematuros foram responsáveis por $47 \%$ das internações, sendo que $15 \%$ dos $\mathrm{RN}$ internados eram recém-nascidos com peso abaixo de 1.500 gramas. Foram excluídos do estudo $448 \mathrm{RN}$ por não preencherem os critérios de inclusão e exclusão estabelecidos, totalizando $2.247 \mathrm{RN}$, que se constituíram na população desse estudo.

Nesse período ocorreram 184 óbitos entre os 2.247 internados, o que resultou em uma letalidade de $8,2 \%$.

A média do número de dias de internação entre todos os RN foi de 19 dias, sendo de 14 dias entre os recém-nascidos que faleceram e 20 dias no grupo dos RN que sobreviveram. O tempo de internação dos recém-nascidos que receberam alta vivos foi de 70 dias para os menores de 1.000 gramas, 47 dias no grupo com peso entre 1.000 e 1.499 gramas, 17 dias para os RN com peso entre 1.500 e 2.499 gramas e (oito) 8 dias para os $\mathrm{RN}$ com peso igual ou maior do que 2.500 gramas.

Entre os recém-nascidos que evoluíram para o óbito, $29 \%$ faleceram no primeiro dia de vida, $30 \%$ entre o segundo e sétimo dia de vida e $30 \%$ do oitavo ao trigésimo dia de vida, com um total de $89 \%$ de óbitos no primeiro mês de vida.

As principais causas de óbito no primeiro dia foram as malformações severas $(31 \%)$, a septicemia $(31 \%)$, a prematuridade extrema $(12 \%)$ e as patologias de placenta e cordão $(8,6 \%)$. A maioria desses recém-nascidos eram prematuros $(84 \%)$ e $58 \%$ apresentavam peso de nascimento menor do que 1.500 gramas.

Os óbitos estratificados por peso de nascimento são apresentados na Tabela 1, mostrando uma relação inversa com o peso. Na comparação com o grupo de peso entre 3.000 e 3.999 gramas, a diferença no número de óbitos mostrou-se estatisticamente significante no grupo de $\mathrm{RN}$ com peso abaixo de 2.000 gramas. A mortalidade dos RN com peso menor do que 1.500 gramas foi de $29,3 \%$ e dos $\mathrm{RN}$ com peso menor do que 1.000 gramas foi de $61,3 \%$, com 5,6\% de mortalidade acima de 1.000 gramas. Os recém-nascidos com baixo peso ao nascer $(<2.500 \mathrm{~g})$ foram responsáveis por $77,7 \%$ de todos os óbitos e apresentaram um risco de morrer 3,6 vezes maior do que os RN com peso igual ou maior do que 2.500 gramas (IC95\%: 2,5-5,2).
Tabela 4

Variáveis associadas com o óbito de recém-nascidos após a análise multivariada. Hospital Geral, Caxias do Sul, Rio Grande do Sul, 1998 a 2004.

\begin{tabular}{lrrr}
\hline Variáveis & OR ajustada (IC95\%) & $p^{*}$ \\
\hline Peso < 2000 gramas & 4,0 & $(2,38-6,83)$ & $<0,001$ \\
Apgar 5' < 4 & 4,2 & $(1,84-9,61)$ & 0,001 \\
Reanimação em sala de parto & $3,6 \quad(2,16-5,87)$ & $<0,001$ \\
Ausência de pré-natal & $2,7 \quad(1,26-5,87)$ & 0,01 \\
Uso de ventilação mecânica & $1,1 \quad(1,04-1,10)$ & $<0,001$ \\
\hline
\end{tabular}

* Valor de $p$ corresponde ao teste qui-quadrado

Tabela 5

Causas básicas de óbito dos recém-nascidos do Hospital Geral. Caxias do Sul, Rio Grande do Sul, 1998 a 2004.

\begin{tabular}{lcr}
\hline Causas & $\mathbf{n}$ & $\%$ \\
\hline Malformações & 47 & 25,6 \\
Hipertensão materna & 33 & 17,9 \\
Prematuridade* & 24 & 13,0 \\
Infecção materna & 22 & 12,0 \\
Ruprema & 16 & 8,7 \\
Patologias da placenta/cordão & 13 & 7,1 \\
Outras & 8 & 4,4 \\
Infecção do RN & 6 & 3,3 \\
Outras doenças maternas & 5 & 2,7 \\
Gestação múltipla & 4 & 2,2 \\
Anóxia & 3 & 1,6 \\
Usuária de drogas & 2 & 1,0 \\
Medicação abortiva & 1 & 0,5 \\
Total & 184 & 100,0 \\
\hline
\end{tabular}

*Prematuridade foi considerada causa básica somente quando não havia uma causa evidente para o desencadeamento do trabalho de parto prematuro.

Em relação à idade gestacional (Tabela 2) verificou-se um risco de morrer estatisticamente significante entre os RN com idade gestacional menor do que 34 semanas, quando comparados com os $\mathrm{RN}$ a termo. O risco de óbito foi 6,8 vezes maior entre os recém-nascidos com idade gestacional menor do que 34 semanas, em comparação aos RN com 34 ou mais semanas de gestação $(\mathrm{p}<0,001)$. Foi constatado, ainda, que o risco de óbito de um recém-nascido prematuro foi 3,8 vezes maior do que o risco de um recém-nascido a termo.

A Tabela 3 apresenta a porcentagem e o risco de 
óbito dos recém-nascidos segundo algumas variáveis maternas e do recém-nascido após a análise univariada.

Para compor o modelo de regressão foram utilizadas as variáveis descritas na tabela 3 que apresentaram significância estatística na análise univariada, com a inclusão do peso de nascimento e idade gestacional. Após a análise multivariada, permaneceram como fatores de risco para o óbito o peso de nascimento menor do que $2.000 \mathrm{~g}$, ausência de pré-natal, a necessidade de reanimação em sala de parto, o Apgar 5' menor do que 4 e a necessidade de ventilação mecânica durante a internação (tabela 4).

A causa básica do óbito está descrita na Tabela 5, podendo ser verificado o grande número de óbitos por malformações. Chama a atenção, também, a grande incidência de hipertensão materna e as infecções maternas como causas desencadeantes do óbito neonatal.

\section{Discussão}

Entre os recém-nascidos que evoluíram para o óbito, $29 \%$ morreram no primeiro dia de vida. Em estudo realizado em Caxias do Sul,7 em 1995, os autores encontraram uma taxa de óbitos no primeiro dia de $47,6 \%$, dado muito semelhante ao encontrado por Philip. 12 Miura et al.13 descreveram em seu estudo $84 \%$ de óbitos nos primeiros sete dias de vida. Os óbitos que ocorrem nos primeiros dias de vida refletem, em geral, a gravidade das patologias, as más condições de nascimento e a presença de um grande número de malformações incompatíveis com a vida.

Entretanto, no presente estudo, encontrou-se um grande número de RN que morreram no primeiro dia por infecção de origem materna, doença que pode ser prevenida por meio de um bom atendimento prénatal. É importante salientar que nesse grupo de RN que morreram no primeiro dia, $16,2 \%$ das mães não fizeram nenhuma consulta na gestação, o que certamente contribuiu para a evolução desfavorável dos RN.

Kilsztajn et al. ${ }^{14}$ verificaram, no estado de São Paulo, que o aumento no número de visitas no prénatal esteve relacionado diretamente com a redução de $\mathrm{RN}$ com retardo do crescimento intra-uterino, prematuridade, nascimento de $\mathrm{RN}$ de baixo peso e mortes neonatais. Menezes et al.15 em Pelotas, encontraram como principais fatores de risco para mortalidade neonatal precoce na cidade de Pelotas, um número de visitas pré-natais menor do que cinco e o baixo peso de nascimento.
Em relação à mortalidade estratificada por peso e idade gestacional, notou-se um aumento estatisticamente significante dos óbitos em recém-nascidos com peso menor do que 2.000 gramas e idade gestacional menor do que 34 semanas. A prematuridade e o baixo peso ao nascer contribuíram com $77,0 \%$ e $77,7 \%$ dos óbitos respectivamente, achados que tem sido encontrados, também, por outros autores 16,17 em diferentes partes do mundo. Shrimpton 18 relata que um aumento de 100 gramas na média do peso de nascimento dos RN de baixo peso está associado com uma redução de 30 a $50 \%$ na mortalidade neonatal, mostrando a importância de programas que visem a melhoria no estado nutricional materno.

Em Porto Alegre, Miura et al. 13 encontraram uma taxa de mortalidade de $34,9 \%$ para os $\mathrm{RN}$ abaixo de 1.500 gramas, incidência um pouco acima da verificada na presente pesquisa(29,3\%). Essa incidência ainda está muito distante dos melhores serviços que apresentam uma mortalidade em torno de $10 \%$ para os RN de muito baixo peso.19,20 O declínio na mortalidade nesses serviços se deve basicamente ao melhor atendimento pré-natal, com a utilização em larga escala do corticóide para a gestante em trabalho de parto prematuro, ao uso de surfactante e aos modernos métodos de ventilação mecânica que melhoraram muito a sobrevida dos RN.

Em relação às variáveis relacionadas com o óbito hospitalar, o peso de nascimento menor do que $2000 \mathrm{~g}$ e o Apgar no 5' menor do que quatro foram as variáveis que apresentaram a maior magnitude de associação com o óbito. Entretanto, é a ausência de pré-natal a variável mais importante, por estar relacionada com um grande número de intercorrências obstétricas e neonatais que resultam no óbito do recém-nascido.

Analisando a causa básica do óbito verificou-se um grande número de afecções maternas $(32,6 \%)$, com predominância para a doença hipertensiva, e de malformações do $\mathrm{RN}$, sendo as cardiopatias as mais freqüentes. Um estudo colaborativo 21 em nove unidades neonatais brasileiras encontrou como fatores de risco para o óbito neonatal, além da prematuridade e do peso de nascimento, a história obstétrica de aborto ou natimorto, asfixia, a doença da membrana hialina, infecções e malformações severas.

Muitas dessas causas estão relacionadas diretamente com as más condições de vida e, portanto, dependem de uma melhor distribuição de renda e um melhor nível educacional da população de baixa renda. Entretanto, verificou-se que um grande número de óbitos poderia ter sido evitado através de uma melhor atenção à gestante no pré-natal. 


\section{Conclusões}

A maioria das mortes ocorreu por problemas relacionados à assistência pré-natal, com um número significativo de gestantes não realizando nenhuma consulta durante a gestação ou iniciando tardiamente

\section{Referências}

1. Ministério da Saúde. Indicadores de mortalidade. Taxa de mortalidade infantil. 2004. Disponível em URL: http://tabnet.datasus.gov.br/cgi/idb2004/c01.htm [2005 jun 4]

2. Ministério da Saúde. Saúde Brasil 2004: uma análise da situação de saúde. Brasília (DF); 2004.

3. Moss W, Darmstadt GL, Marsh DR, Black RE, Santosham M. Research priorities for the reduction of perinatal and neonatal morbidity and mortality in developing country communities. J Perinatol. 2002; 22: 484-95.

4. Bercini LO. Mortalidade neonatal de residentes em localidade urbana da região Sul do Brasil. Rev Saúde Pública 1994; 28: 38-45.

5. Victora CG, Barros FC, Vaughan JP. Epidemiologia da desigualdade. São Paulo: Hucitec; 1998.

6. Menezes AMB, Barros FC, Victora CG. Mortalidade perinatal em duas coortes de base populacional no Sul do Brasil: tendências e diferenciais. Cad Saúde Pública 1996; 12: 33-41.

7. Araújo BF, Bozzetti MC, Tanaka ACA. Mortalidade neonatal precoce no município de Caxias do Sul: um estudo de coorte. J Pediatr (Rio J) 2000; 76: 200-6.

8. Arias E, MacDorman MF, Strobino DM, Guyer B. Annual summary of vital ststistics-2002. Pediatrics 2003; 112: 1215-30.

9. Bercini LO. Neonatal mortality in residents of an urban locality in the Southern region of Brazil. Rev Saude Publica 1994; 28: 38-45.

10. Albacar E, Borrell C. Trends in infant mortality in Barcelona, 1983-1998. Gac Sanit 2004; 18: 24-31.

11. OMS (Organização Mundial da Saúde). Classificação Estatística Internacional de Doenças e Problemas relacionados à Saúde (CID 10). São Paulo: Universidade de São Paulo, 1997. o acompanhamento pré-natal. Reduzir as mortes evitáveis no período neonatal é um desafio que precisa ser enfrentado pelos neonatologistas, obstetras e pelo setor público, através de políticas públicas voltadas para essa faixa etária, que hoje é responsável por $75 \%$ dos óbitos infantis.
12. Philip AGS. Neonatal mortality rate: is further improvement possible? J Pediatr 1995; 126: 427-33.

13. Miura E, Failace LH, Fiori H. Mortalidade perinatal e neonatal no Hospital de Clínicas de Porto Alegre. Rev Ass Med Brasil 1997; 43: 35-9.

14. Kilsztajn S, Rossbach A, Carmo MS, Sugahara GT. Prenatal care, low birthweight and prematurity in São Paulo State, Brazil, 2000. Rev Saude Publica 2003; 37: 303-10.

15. Menezes AM, Barros FC, Victora CG, Tomasi E, Halpern $\mathrm{R}$, Oliveira AL. Risk factors for perinatal mortality in Pelotas, a Southern city of Brazil,1993. Rev Saúde Pública 1998; 32: 209-16.

16. Kaushik SL, Parmar VR, Grover N, Kaushik R. Neonatal mortality rate: relationship to birth weight and gestational age. Indian J Pediatrics 1998; 65: 429-33.

17. Gould JB, Benitz WE, Liu H. Mortality and time to death in very low birth weight infants: California, 1987 and 1993. Pediatrics 2000; 105: E37.

18. Shrimpton R. Preventing low birthweight and reduction of child mortality. Trans R Soc Trop Med Hyg. 2003; 97: 3942.

19. Richardson DK, Gray JE, Gortmaker SL, Goldmann DA, Pursley DM, McCormick MC. Declining severity adjusted mortality:evidence of improving neonatal intensive care. Pediatrics 1998;102: 893-9.

20. Wen SW, Kramer MS, Liu S, Dzakpasu S, Sauve R. Infant mortality by gestational age and birth weight in Canadian provinces and territories, 1990-1994 births. Chronic Dis Can 2000; 21: 14-22.

21. Zullini MT, Bonati M, Sanvito E. Survival at nine neonatal intensive care units in Sao Paulo, Brazil. Paulista Collaborative Group on Neonatal Care. Rev Panam Salud Publica 1997; 2: 303-9. 